\title{
Equatorial circular orbits in Kerr-Newman-de Sitter spacetimes
}

\author{
Petr Slanýa ${ }^{\mathrm{a}}$ Zdeněk Stuchlík ${ }^{\mathrm{b}}$ \\ Research Centre of Theoretical Physics and Astrophysics, Institute of Physics, Silesian University in Opava, Bezručovo nám. 13, 74601 Opava, \\ Czech Republic
}

Received: 15 April 2020 / Accepted: 10 June 2020 / Published online: 29 June 2020

(C) The Author(s) 2020

\begin{abstract}
Circular motion of test particles in the equatorial plane of the Kerr-Newman-de Sitter (KNdS) spacetime is analyzed for both black-hole and naked-singularity backgrounds. We present relations for specific energy, specific angular momentum and Keplerian angular velocity of a particle on equatorial circular orbit, and discuss criteria for the existence of such orbits giving limits on spacetime parameters. The orientation of motion along circular orbits is discussed from the point of view of locally non-rotating frames. Finally, we discuss the stability of circular motion against radial perturbations and determine limits on the existence of stable circular orbits, as well as the structure of stability regions in KNdS spacetimes.
\end{abstract}

\section{Introduction}

More than two decades of astronomical observations, including high-redshift supernovae, cosmic microwave background radiation and large-scale structure, indicate convincingly accelerated expansion of the Universe [1-5]. As commonly accepted hypothesis, the dark energy, demonstrating gravitational repulsion, was proposed to cause this phenomenon. Detailed analyses of various data show that the dark energy behaves like the vacuum energy, which, in relativistic cosmology, is represented by a repulsive cosmological constant $\Lambda>0[6,7]$. Its current value, being in accordance with astronomical observations, is of the order $10^{-52} \mathrm{~m}^{-2}$. Important alternatives to the vacuum energy are represented by a variety of quintessential fields [8-10]. On the other hand, the recent accelerated expansion of our observable Universe can be explained also in the non-standard way, e.g. in the framework of inhomogeneous or anisotropic cosmologies [11-13].

Here we assume the case of repulsive cosmological constant. Due to this fact, the structure of black-hole (naked-

\footnotetext{
${ }^{a}$ e-mail: petr.slany@physics.slu.cz (corresponding author)

b e-mail: zdenek.stuchlik@physics.slu.cz
}

singularity) solutions of Einstein equations of General Relativity is substantially modified in comparison with the $\Lambda=0$ case, as these solutions contain another, so-called cosmological, event horizon, with a dynamic region behind it $[14,15]$. For $\Lambda \sim 10^{-52} \mathrm{~m}^{-2}$, the cosmological horizon is located at the distance $r_{\mathrm{c}} \sim 10^{26} \mathrm{~m}$, comparable with the current Hubble radius of the Universe. Such a value of $r_{\mathrm{c}}$ is relevant for all astronomical black holes, as their masses are well below $10^{11} \mathrm{M}_{\odot}$. We should stress that the cosmological horizon is important for cosmology and related problems, but it is irrelevant for astrophysics of black holes. However, various studies show that for astrophysical processes, another radius related to the cosmic repulsion could be relevant. This is the static (or turnaround) radius [16-18], depending on the central mass $M$ and cosmological constant $\Lambda$ as $r_{\mathrm{s}}=\sqrt[3]{3 G M / \Lambda c^{2}}$. At the static radius, the gravitational attraction caused by the central mass is just compensated by the cosmic repulsion described by the cosmological constant. It has been shown that the static radius gives an upper limit on an extension of disk-like structures around black holes, coinciding with dimensions of large galaxies with central supermassive black holes [19-22], and gives also a natural limit on extension of bound systems in an expanding universe with $\Lambda>0$ [23-28].

Till now, studies of the black-hole (naked-singularity) backgrounds with $\Lambda>0$ were mainly concentrated on uncharged Schwarzschild-de Sitter and Kerr-de Sitter spacetimes [16,29-37]. However, theoretical considerations, together with recent observations of structures near Sgr A* by the GRAVITY experiment [38], indicate possible presence of a small electric charge of central supermassive black hole $[39,40]$. Therefore, it could be interesting to study the combined effect of cosmic repulsion and electromagnetic fields on the black-hole astrophysics. The simplest case would be, of course, their influence on the motion of uncharged matter. In the case of non-rotating Reissner-Nordström-de Sitter black holes, the circular motion of test particles and photons was studied in [41], where the naked-singularity background 
was analyzed too. Here we include also the influence of black hole (naked singularity) rotation.

More precisely, we concentrate on circular geodesics in the equatorial plane of the Kerr-Newman-de Sitter (KNdS) spacetime, which incorporates both the gravity of a charged rotating black hole (naked singularity) and the cosmic repulsion, and corresponds to the most general asymptotically de Sitter black-hole solution of Einstein's General Relativity. Due to this fact, such an analysis can also have a significant theoretical meaning.

\section{The background}

The KNdS solution describes spacetime geometry of a rotating and electrically charged black hole (naked singularity) in the universe with non-zero cosmological constant. In the well-known Boyer-Lindquist coordinates $(t, r, \theta, \varphi)$ and geometrical units ( $c=G=1)$, the spacetime geometry is described by the line element [14]

$$
\begin{aligned}
\mathrm{d} s^{2}= & -\frac{\Delta_{r}}{I^{2} \rho^{2}}\left(\mathrm{~d} t-a \sin ^{2} \theta \mathrm{d} \varphi\right)^{2} \\
& +\frac{\Delta_{\theta} \sin ^{2} \theta}{I^{2} \rho^{2}}\left[a \mathrm{~d} t-\left(r^{2}+a^{2}\right) \mathrm{d} \varphi\right]^{2} \\
& +\frac{\rho^{2}}{\Delta_{r}} \mathrm{~d} r^{2}+\frac{\rho^{2}}{\Delta_{\theta}} \mathrm{d} \theta^{2},
\end{aligned}
$$

where

$$
\begin{aligned}
\Delta_{r} & =r^{2}-2 M r+a^{2}+e^{2}-\frac{1}{3} \Lambda r^{2}\left(r^{2}+a^{2}\right), \\
\Delta_{\theta} & =1+\frac{1}{3} \Lambda a^{2} \cos ^{2} \theta, \\
I & =1+\frac{1}{3} \Lambda a^{2} \\
\rho^{2} & =r^{2}+a^{2} \cos ^{2} \theta .
\end{aligned}
$$

It depends on four parameters, $M, a, e$, and $\Lambda$, corresponding, respectively, to the mass, spin and electric charge of a central black hole/naked singularity, and to the cosmological constant. Together with the gravitational field there is also related electromagnetic field described by the 4-potential

$A_{\mu}=-\frac{e r}{I \rho^{2}}\left(\delta_{\mu}^{t}-a \sin ^{2} \theta \delta_{\mu}^{\varphi}\right)$.

For $a=0$, the relations (1)-(6) describe geometry and electrostatic field of the Reissner-Nordström-de Sitter spacetime. In the uncharged case, we can distinguish the Kerrde Sitter $(e=0, a \neq 0)$ or Schwarzschild-de Sitter $(e=0, a=0)$ spacetimes.

In order to simplify relations, instead of the cosmological constant $\Lambda$ we use a dimensionless cosmological parameter $y$, $y=\frac{1}{3} \Lambda M^{2}$.

Almost all quantities are scaled with the mass $M$, therefore it is useful to fix the mass parameter putting $M=1$. Remaining three parameters $(a, e, y)$ are still free and limits of their values will be a part of discussion in our analysis.

\section{Horizons}

Event horizons of the KNdS spacetime correspond to null hypersurfaces of $r=$ const determined by the condition $g^{r r}=0$, i.e. $\Delta_{r}=0$, which can be rewritten into the form

$y=y_{\mathrm{h}}(r ; a, e) \equiv \frac{r^{2}-2 r+a^{2}+e^{2}}{r^{2}\left(r^{2}+a^{2}\right)}$.

Detailed analysis of the function $y_{\mathrm{h}}(r ; a, e)$ can be found in [42], here we summarize only the most important results.

In all $\mathrm{KNdS}$ spacetimes $(y>0)$, there is always a cosmological horizon located at $r=r_{\mathrm{c}}$. If the values of spin and charge of the central mass allow existence of black holes, the function $y_{\mathrm{h}}(r ; a, e)$ has two local extrema-minimum $y_{\mathrm{h}, \text { min }}$ and maximum $y_{\mathrm{h}, \max }$ which is always positive. Then for cosmological parameter $y_{\mathrm{h}, \text { min }}<y<y_{\mathrm{h} \text {, max }}$, the spacetime contains also two black-hole horizons, the inner one at $r=r_{-}$ and the outer one at $r=r_{+}$where $r_{-}<r_{+}<r_{\mathrm{c}}$. In regions $r_{-}<r<r_{+}$and $r>r_{\mathrm{c}}$ the spacetime is dynamic, forbidding the existence of stationary observers at $r=$ const orbits. For $0<a^{2}+e^{2}<1$, the local minimum $y_{\mathrm{h}, \min }$ is negative, and for cosmological parameter $y_{\mathrm{h}, \min }<y<0$ the spacetime contains only two horizons $r_{ \pm}$corresponding to blackhole horizons of the Kerr-Newman-anti-de Sitter (KNadS) black hole. Independently of the sign of $y_{\mathrm{h}, \min }$, for $y=y_{\mathrm{h}, \min }$ both black-hole horizons coincide, $r_{+}=r_{-}$, and the spacetime geometry describes extreme $\mathrm{KN}(\mathrm{a}) \mathrm{dS}$ black hole. Other cases correspond to naked-singularity spacetimes. Behavior of the function $y_{\mathrm{h}}(r ; a, e)$ for various values of the rotational and charge parameters is presented in Fig. 1.

Extrema of the function $y_{\mathrm{h}}(r ; a, e)$ are given by the condition

$e^{2}=e_{\mathrm{ex}(\mathrm{h})}^{2}(r ; a) \equiv \frac{r\left(3 r^{2}+a^{2}\right)-\left(r^{2}+a^{2}\right)^{2}}{2 r^{2}+a^{2}}$,

where only non-negative values of the function $e_{\mathrm{ex}(\mathrm{h})}^{2}(r ; a)$ are physically relevant. The analysis of its behavior in dependence of the rotational parameter $a$ shows that $e_{\mathrm{ex}(\mathrm{h})}^{2} \geq 0$ only for $a^{2} \in\left\langle 0, a_{\text {crit }}^{2}\right\rangle$, where $a_{\text {crit }}^{2}=3(2 \sqrt{3}+3) / 16 \doteq$ 1,21202 . The function $e_{\mathrm{ex}(\mathrm{h})}^{2}(r ; a)$ is for $r>0$ strictly concave, possessing one local maximum which value depends on the parameter $a$ as 


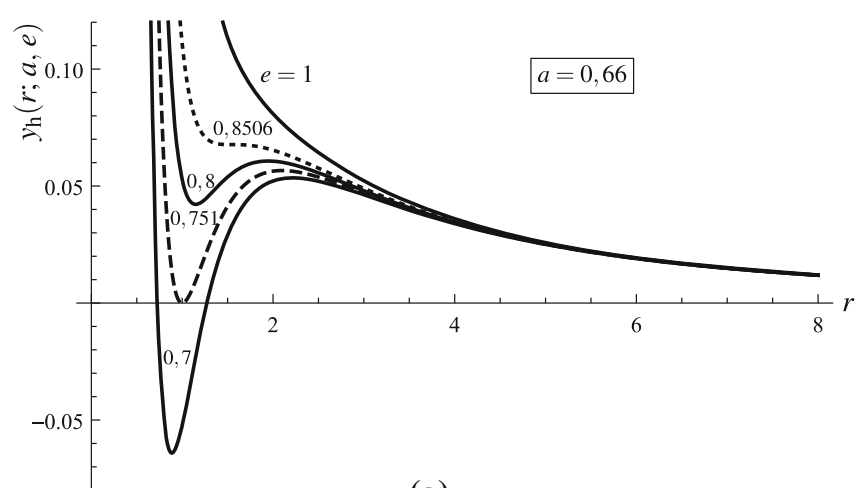

(a)

Fig. 1 Profiles of the function $y_{\mathrm{h}}(r ; a, e)$ for a fixed value of the parameter $a$ and various values of the parameter $e$, $\mathbf{b}$ fixed value of the parameter $e$ and various values of the parameter $a$. Dashed profiles

$$
\begin{aligned}
e_{\mathrm{ex}(\mathrm{h}), \max }^{2}(a)= & \frac{3}{8}\left[F^{2}(a)\left(1+a^{2}-\sqrt{a^{2}\left(a^{2}+2\right)}\right)\right. \\
& \left.+F(a)-\frac{8}{3} a^{2}+1\right] \\
F(a)= & \left(1+a^{2}+\sqrt{a^{2}\left(a^{2}+2\right)}\right)^{1 / 3} .
\end{aligned}
$$

Function $e_{\mathrm{ex}(\mathrm{h}) \text {,max }}^{2}(a)$ is monotonically decreasing in the interval $a \in\left\langle 0, a_{\text {crit }}\right\rangle$, reaching its minimal value for $a=$ $a_{\text {crit }}$. Therefore the maximal value, denoted as $e_{\text {crit }}^{2}$, this function reaches for $a=0$. From relation (10) it is clear that $e_{\text {crit }}^{2}=9 / 8$.

Previous analysis of the function $e_{\mathrm{ex}(\mathrm{h})}^{2}(r ; a)$ shows that the KNdS spacetime contains a black hole only if its parameters do not exceed certain critical values $0 \leq a^{2} \leq a_{\text {crit }}^{2}$, $0 \leq e^{2} \leq e_{\text {crit }}^{2}$, where the higher is the value of the charge parameter $e$ from given interval, the lower is the maximal value of the rotational parameter $a$ admitting a black hole, and vice versa. For limiting values both extrema of the function $y_{\mathrm{h}}(r ; a, e)$ coincide in an inflection point determining, for a given $e$ and $a$, a limiting value of the cosmological parameter $y$ admitting the existence of black holes. Analysis of the function $y_{\mathrm{h}}(r ; a, e)$ shows, see also [42], that the maximal value of the cosmological parameter $y$ admitting the existence of KNdS black hole coincides with the maximal value for extreme Reissner-Nordström-de Sitter black hole [43], $y_{\text {crit }}=2 / 27$. It should be noted that the maximal value of the cosmological parameter admitting the existence of Kerrde Sitter black hole is $y_{\mathrm{c}(\mathrm{KdS})}=16 /(2 \sqrt{3}+3)^{3}$, while for the Schwarzschild-de Sitter black hole it is $y_{\mathrm{c}(\mathrm{SdS})}=1 / 27$, see $[29,30]$.

\section{Equations of motion}

Motion of a test charged particle with mass $m$ and electric charge $q$ can be the most effectively analyzed by the

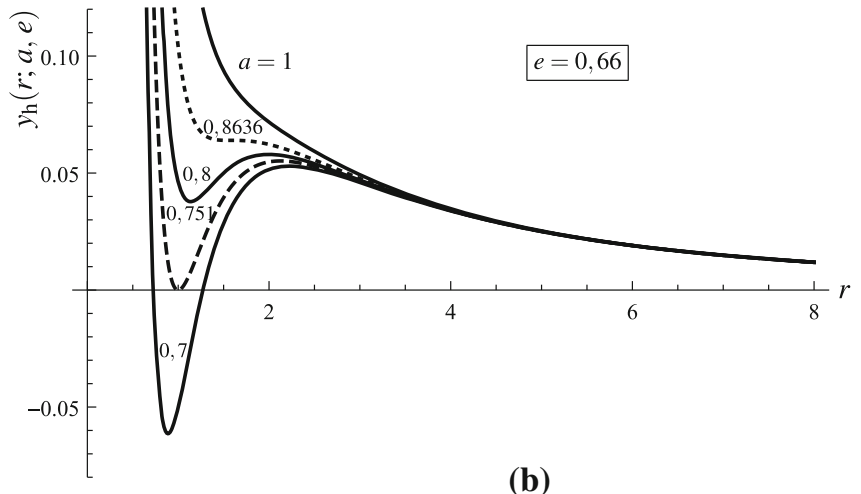

correspond to the case where $a^{2}+e^{2}=1$, dotted profiles correspond to situation in which both local extrema coincide in an inflex point

Hamilton-Jacobi formalism. The corresponding HamiltonJacobi equation [44],

$-\frac{\partial S}{\partial \lambda}=\frac{1}{2} g^{\mu \nu}\left(\frac{\partial S}{\partial x^{\mu}}-q A_{\mu}\right)\left(\frac{\partial S}{\partial x^{\nu}}-q A_{\nu}\right)$,

for the principal Hamilton function $S(\lambda, t, \varphi, r, \theta)$ takes, in the $\mathrm{KNdS}$ spacetime, the form

$$
\begin{aligned}
-2 \frac{\partial S}{\partial \lambda}= & -\frac{1}{\rho^{2} \Delta_{r}}\left[I\left(r^{2}+a^{2}\right) \frac{\partial S}{\partial t}+I a \frac{\partial S}{\partial \varphi}+e q r\right]^{2} \\
& +\frac{1}{\rho^{2} \Delta_{\theta} \sin ^{2} \theta}\left[I a \sin ^{2} \theta \frac{\partial S}{\partial t}+I \frac{\partial S}{\partial \varphi}\right]^{2} \\
& +\frac{\Delta_{r}}{\rho^{2}}\left(\frac{\partial S}{\partial r}\right)^{2}+\frac{\Delta_{\theta}}{\rho^{2}}\left(\frac{\partial S}{\partial \theta}\right)^{2} ;
\end{aligned}
$$

$\lambda$ is an affine parameter connected with particle's proper time $\tau$ by the relation $\lambda=\tau / m$ so that

$g_{\mu \nu} \frac{\mathrm{d} x^{\mu}}{\mathrm{d} \lambda} \frac{\mathrm{d} x^{\nu}}{\mathrm{d} \lambda}=-m^{2}$,

where $m^{2}$ can be considered as the first constant of motion. Both the spacetime and the electromagnetic field are stationary and axially symmetric, thus the corresponding time and azimuthal canonical momenta $p_{\mu}=\partial S / \partial x^{\mu}$ are another constants of motion: $p_{t}=-\mathscr{E}, p_{\varphi}=\Phi .{ }^{1}$ As was shown by B. Carter in the case of Kerr-Newman spacetime [14,45], a solution of the Hamilton-Jacobi equation can be written in a separated form,

$S=\frac{1}{2} m^{2} \lambda-\mathscr{E} t+\Phi \varphi+S_{r}(r)+S_{\theta}(\theta)$,

\footnotetext{
${ }^{1}$ In asymptotically flat Kerr-Newman spacetime $(\Lambda=0)$ these constants correspond, respectively, to energy and axial angular momentum of a particle at infinity but in asymptotically de Sitter background such interpretation is not possible. However despite this fact we will still call these quantities "energy" and "angular momentum".
} 
where $S_{r}$ and $S_{\theta}$ are some functions of their variables, leading to the fourth constant of motion $\mathscr{K}$ and enabling to find a complete set of first integrals of motion. The same technique holds also in the KNdS spacetime for which

$$
S_{r}=\int^{r} \frac{1}{\Delta_{r}} \sqrt{R(r)} \mathrm{d} r, \quad S_{\theta}=\int^{\theta} \frac{1}{\Delta_{\theta}} \sqrt{W(\theta)} \mathrm{d} \theta,
$$

where

$$
\begin{aligned}
R(r) & =P_{r}^{2}-\Delta_{r}\left(m^{2} r^{2}+\mathscr{K}\right), \\
P_{r} & =I \mathscr{E}\left(r^{2}+a^{2}\right)-I a \Phi-e q r, \\
W(\theta) & =\Delta_{\theta}\left(\mathscr{K}-m^{2} a^{2} \cos ^{2} \theta\right)-\frac{P_{\theta}^{2}}{\sin ^{2} \theta}, \\
P_{\theta} & =I\left(a \mathscr{E} \sin ^{2} \theta-\Phi\right) .
\end{aligned}
$$

Applying the Hamilton-Jacobi method, we obtain the integral form of equations of motion:

$$
\begin{aligned}
\int & \frac{\mathrm{d} r}{\sqrt{R}}=\int^{\theta} \frac{\mathrm{d} \theta}{\sqrt{W}}, \\
\lambda= & \int^{r} \frac{r^{2}}{\sqrt{R}} \mathrm{~d} r+\int^{\theta} \frac{a^{2} \cos ^{2} \theta}{\sqrt{W}} \mathrm{~d} \theta, \\
t= & \int^{r} \frac{I\left(r^{2}+a^{2}\right)\left[I \mathscr{E}\left(r^{2}+a^{2}\right)-I a \Phi-e q r\right]}{\Delta_{r} \sqrt{R}} \mathrm{~d} r \\
& -\int^{\theta} \frac{I^{2} a\left(a \mathscr{E} \sin ^{2} \theta-\Phi\right)}{\Delta_{\theta} \sqrt{W}} \mathrm{~d} \theta, \\
\varphi= & \int^{r} \frac{I a\left[I \mathscr{E}\left(r^{2}+a^{2}\right)-I a \Phi-e q r\right]}{\Delta_{r} \sqrt{R}} \mathrm{~d} r \\
& -\int^{\theta} \frac{I^{2}\left(a \mathscr{E} \sin ^{2} \theta-\Phi\right)}{\Delta_{\theta} \sqrt{W} \sin ^{2} \theta} \mathrm{d} \theta .
\end{aligned}
$$

However, for subsequent discussion, their differential form following directly from the integral form is more useful [16]:

$\rho^{2} \frac{\mathrm{d} r}{\mathrm{~d} \lambda}= \pm \sqrt{R(r)}$

$\rho^{2} \frac{\mathrm{d} \theta}{\mathrm{d} \lambda}= \pm \sqrt{W(\theta)}$,

$\rho^{2} \frac{\mathrm{d} t}{\mathrm{~d} \lambda}=\frac{I\left(r^{2}+a^{2}\right) P_{r}}{\Delta_{r}}-\frac{I a P_{\theta}}{\Delta_{\theta}}$,

$\rho^{2} \frac{\mathrm{d} \varphi}{\mathrm{d} \lambda}=\frac{I a P_{r}}{\Delta_{r}}-\frac{I P_{\theta}}{\Delta_{\theta} \sin ^{2} \theta}$.

Carter's equations (24)-(27) describe motion of a charged test particle in the KNdS spacetime. Further we focus our attention to circular motion of test uncharged particles in the equatorial plane $\theta=\pi / 2$, i.e. to equatorial circular timelike geodesics.

\section{Circular geodesics in the equatorial plane}

If the motion of a test particle is restricted to the equatorial plane $\theta=\pi / 2$, then $\mathrm{d} \theta / \mathrm{d} \lambda=0$, and from equation (25) follows that the constants of motion are connected by the relation

$\mathscr{K}=I^{2}(a \mathscr{E}-\Phi)^{2}$.

Now we define specific energy $E$ and specific angular momentum $L$ of a particle by relations

$E \equiv \frac{I \mathscr{E}}{m}, \quad L \equiv \frac{I \Phi}{m}$.

Then the Eq. (24), describing radial motion, for test uncharged particle moving in the equatorial plane takes the form

$r^{2} \frac{\mathrm{d} r}{\mathrm{~d} \tau}= \pm \sqrt{\widetilde{R}(r)}$

where

$\widetilde{R}(r)=\left[E\left(r^{2}+a^{2}\right)-a L\right]^{2}-\Delta_{r}\left[r^{2}+(a E-L)^{2}\right]$.

In order to simplify description and further calculations, it is useful to introduce another axial parameter

$X \equiv L-a E$,

giving

$\widetilde{R}(r)=\left(E r^{2}-a X\right)^{2}-\Delta_{r}\left(r^{2}+X^{2}\right)$.

Equation (30) governs the radial motion of uncharged test particles in the equatorial plane. Taking its square, or simply from the reality condition that any relation under a square root has to be non-negative, the test particle motion is allowed only in the region where $\widetilde{R}(r) \geq 0$. The radial function $\widetilde{R}(r)$ is a quadratic trinomial for the specific energy $E$. Therefore, the motion of a particle with given specific energy $E$ and axial parameter $X$ is allowed only in that regions where $E \geq V_{+}(r ; X)$, or $E \leq V_{-}(r ; X)$; equalities correspond to turning points of radial motion and the functions

$V_{ \pm}(r ; X)=\frac{1}{r^{2}}\left[a X \pm \sqrt{\Delta_{r}\left(r^{2}+X^{2}\right)}\right]$

correspond to the roots of the equation $\widetilde{R}(r)=0$. If we restrict only on particles in so-called "positive-root states" [44], i.e. particles on future directed trajectories, then from the condition $\mathrm{d} t / \mathrm{d} \tau>0$ follows that their energy must satisfy the inequality

$E>\frac{a X}{r^{2}}\left(1-\frac{\Delta_{r}}{r^{2}+a^{2}}\right)$.

Comparing the condition (35) with behavior of functions $V_{ \pm}(r ; X)$ reveals that for such particles only the function $V_{+}$is relevant. In that sense, $V_{+}(r ; X)$ plays a role of the effective potential governing the radial motion of uncharged test particles in the equatorial plane of the KNdS spacetime. 
Fig. 2 Radial profiles of specific energy and specific angular momentum of test particles moving on equatorial circular orbits around $\mathrm{KNdS}$ black holes with cosmological parameter $y=10^{-4}$. Values of corresponding rotational and charge parameters $(a, e)$ are assigned to each curve separately. a, b Correspond to the plus-family orbits, while the c, $\mathbf{d}$ correspond to the minus-family orbits

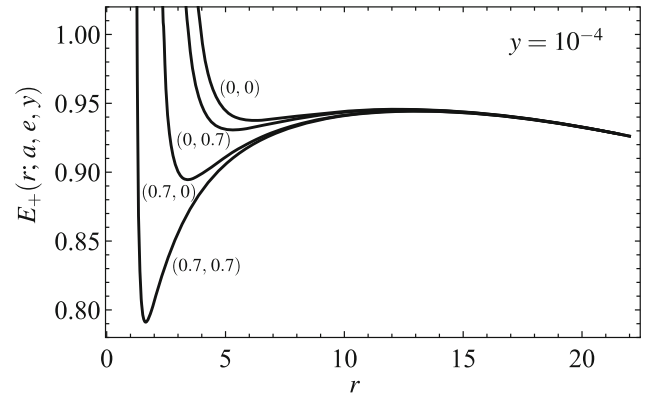

(a)

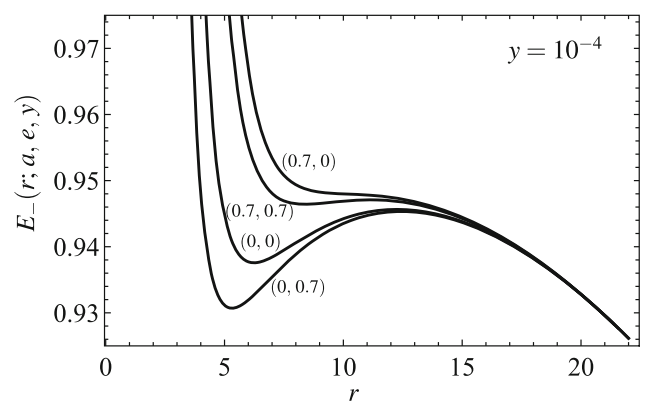

(c)

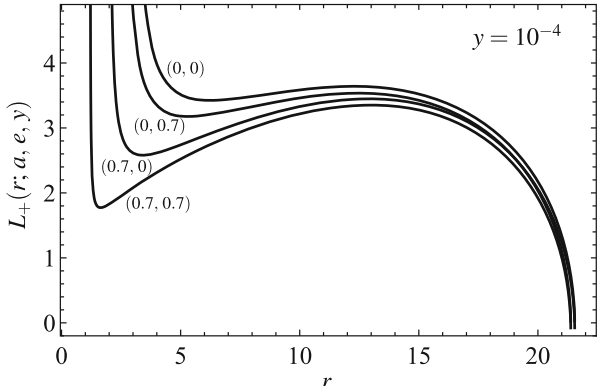

(b)

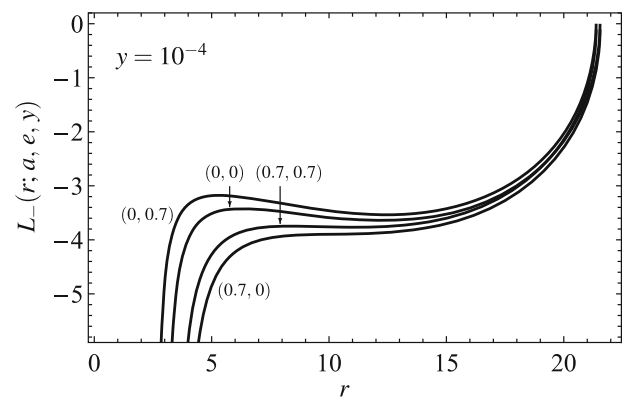

(d)
Extrema of the function $V_{+}(r ; X)$ in terms of radial coordinate $r$ determine positions of equatorial circular geodesics and, subsequently, values of corresponding constants of motion. Equivalently, circular geodesics are given by conditions

$\widetilde{R}(r)=0, \quad \frac{\mathrm{d} \widetilde{R}}{\mathrm{~d} r}=0$,

which must be both satisfied. Applying the same procedure for elimination of variables as in the case of Kerr-de Sitter background [30], the specific energy and specific angular momentum of an uncharged test particle on circular orbit (geodesic) in the equatorial plane of the KNdS spacetime are given by relations

$$
\begin{aligned}
& E_{ \pm}(r ; a, e, y) \\
& =\frac{1-\frac{2}{r}+\frac{e^{2}}{r^{2}}-y\left(r^{2}+a^{2}\right) \pm a \sqrt{\frac{1}{r^{3}}-\frac{e^{2}}{r^{4}}-y}}{\left[1-\frac{3}{r}+2 \frac{e^{2}}{r^{2}}-y a^{2} \pm 2 a \sqrt{\frac{1}{r^{3}}-\frac{e^{2}}{r^{4}}-y}\right]^{1 / 2}}, \\
& L_{ \pm}(r ; a, e, y) \\
& =\frac{-\frac{2 a}{r}+\frac{a e^{2}}{r^{2}}-y a\left(r^{2}+a^{2}\right) \pm\left(r^{2}+a^{2}\right) \sqrt{\frac{1}{r^{3}}-\frac{e^{2}}{r^{4}}-y}}{\left[1-\frac{3}{r}+2 \frac{e^{2}}{r^{2}}-y a^{2} \pm 2 a \sqrt{\frac{1}{r^{3}}-\frac{e^{2}}{r^{4}}-y}\right]^{1 / 2}} .
\end{aligned}
$$

As in other Kerr-like spacetimes, there are two families of orbits - the plus-family and the minus-family - correspond- ing to upper and lower signs in relations (37) and (38). Note that given relations for specific energy and specific angular momentum are valid also for motion of an uncharged test particle on equatorial circular geodesic in the KNadS spacetime. Further discussion, however, we will continue only for the case of a repulsive cosmological constant, i.e. for the cosmological parameter $y>0$. Characteristic behavior of radial profiles of specific energy and specific angular momentum, showing the change of constants of motion $E$ and $L$ during the transition from one orbit to another one, is presented for appropriately chosen spacetime parameters in Figs. 2 and 3 . Importance of local extrema of the functions $E_{ \pm}(r ; a, e, y)$ and $L_{ \pm}(r ; a, e, y)$ is related to stability of orbits against radial perturbations, and will be discussed later.

\subsection{Existence of equatorial circular geodesics}

Reality conditions

$$
\begin{aligned}
& \frac{1}{r^{3}}-\frac{e^{2}}{r^{4}}-y \geq 0, \\
& 1-\frac{3}{r}+2 \frac{e^{2}}{r^{2}}-y a^{2} \pm 2 a \sqrt{\frac{1}{r^{3}}-\frac{e^{2}}{r^{4}}-y}>0,
\end{aligned}
$$

following from the relations (37) and (38) for specific energy and specific angular momentum, mean that equatorial circular geodesics exist only in those regions of spacetime where both conditions are satisfied.

The first condition (39) can be rewritten to the form

$y \leq y_{\mathrm{s}}(r ; e) \equiv \frac{r-e^{2}}{r^{4}}$, 
Fig. 3 Radial profiles of specific energy and specific angular momentum of test particles moving on equatorial circular orbits around $\mathrm{KNdS}$ naked singularities with spacetime parameters shown in each panel. Values of rotational and charge parameters $(a, e)$ are assigned to each curve separately. a, b Correspond to the plus-family orbits, while the c, $\mathbf{d}$ correspond to the minus-family orbits

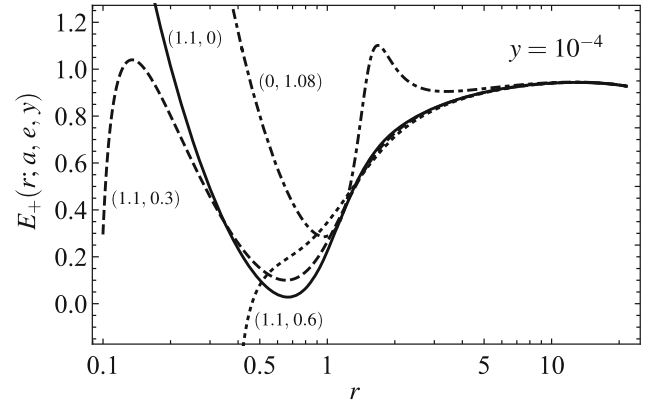

(a)

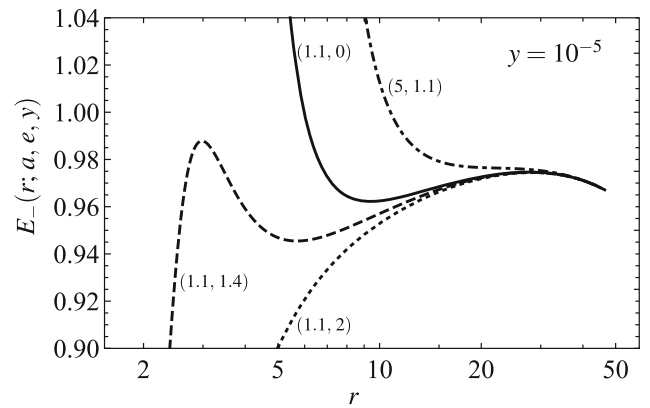

(c)

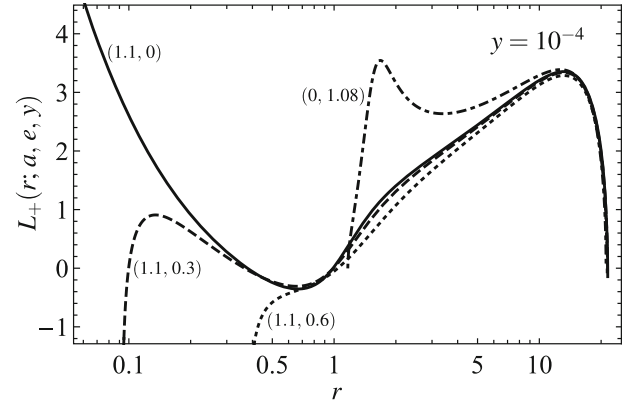

(b)

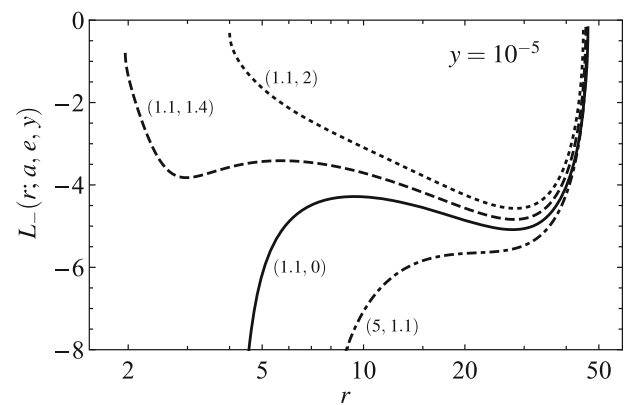

(d) where the function $y_{\mathrm{s}}(r ; e)$ determines so called static radii of the KNdS spacetime. Since the static radii are determined independently of the rotational parameter $a$, these are the same as in the Reissner-Nordström-de Sitter spacetime [41]. Static radius is an orbit where freely falling observer has non-zero time-component $P^{t}$ of its 4-momentum only; other (space-)components $P^{i}$ are zero there which can be seen directly from the geodesic Eqs. (24)-(27) for $y=y_{\mathrm{s}}(r ; e)$ and $\theta=\pi / 2$.

Function $y_{\mathrm{s}}(r ; e)$ has one local extreme - maximum - at $r=r_{\mathrm{ex}(\mathrm{s})} \equiv 4 e^{2} / 3$, giving the value $y_{\mathrm{ex}(\mathrm{s})}=27 / 256 e^{6}$. Moreover, for $r \rightarrow 0$, the function $y_{\mathrm{s}}(r ; e) \rightarrow-\infty$, while for $r \rightarrow \infty$, the function $y_{\mathrm{s}}(r ; e) \rightarrow 0$. Therefore, in $\mathrm{KNdS}$ spacetimes with cosmological parameter $y<y_{\mathrm{ex}(\mathrm{s})}$, there are two static radii, $r_{\mathrm{s} 1}$ a $r_{\mathrm{s} 2}$, where $r_{\mathrm{s} 1}<r_{\mathrm{s} 2}$, but not always both lie in physically important stationary regions of spacetime. In black-hole spacetimes with charge parameter $e^{2} \leq 1$ and rotational parameter $a^{2}<e^{2}\left(1-e^{2}\right)$, the inner radius $r_{\mathrm{s} 1}$ is located in dynamic region between black-hole horizons, $r_{-}<r_{\mathrm{s} 1}<r_{+}$, and thus, in fact, does not influence the existence of circular orbits. If $a^{2}>e^{2}\left(1-e^{2}\right.$ ) (for $\left.e^{2} \leq 1\right)$, and for $e^{2}>1$ there are KNdS black holes, for which $r_{\mathrm{s} 1}<r_{-}$, i.e. it lies in a stationary region inside the black hole. Intersections of functions $y_{\mathrm{h}}(r ; a, e)$ and $y_{\mathrm{s}}(r ; e)$ reveal that in black-hole spacetimes with sufficiently large value of cosmological parameter $y \lesssim y_{\mathrm{h} \text {, max }} \sim y_{\text {crit }}=2 / 27$, the outer radius $r_{\mathrm{s} 2}$ lies behind the cosmological horizon $r_{\mathrm{c}}$ and, thus, has no relevance for the existence of circular orbits. Nevertheless, in most of black-hole spacetimes the outer static radius lies in a stationary region between the black hole and the cosmological horizon, $r_{+}<r_{\mathrm{s} 2}<r_{\mathrm{c}}$. Analogous analysis in the case of naked singularities reveals that in a stationary region $0<r<r_{\mathrm{c}}$, there can be one, two, or even no static radii. The better insight into possible mutual positions of spacetime horizons and static radii gives Fig. 4 showing behavior of functions $y_{\mathrm{h}}(r ; a, e)$ a $y_{\mathrm{s}}(r ; e)$ for various values of spacetime parameters $a$ and $e$. Note that in uncharged Schwarzschild-de Sitter and Kerr-de Sitter spacetimes, there is always only one static radius located at the position $r_{\mathrm{s}}=y^{-1 / 3}[16,29,30]$.

Condition (40) corresponds to limits on the existence of circular orbits given by orbits of photons. In KNdS spacetimes, their location is given by the relation

$$
\begin{aligned}
y & =y_{\mathrm{ph}}(r ; a, e) \\
& \equiv \frac{-\left(r^{2}+3 r-2 e^{2}\right)+2 \sqrt{r\left(3 r^{2}+a^{2}\right)-e^{2}\left(2 r^{2}+a^{2}\right)}}{a^{2} r^{2}} .
\end{aligned}
$$

Detailed analysis of the function $y_{\mathrm{ph}}(r ; a, e)$ can be found in [42], here we are going to present only its basic characteristics.

In black-hole spacetimes, the function $y_{\mathrm{ph}}(r ; a, e)$ has three local extrema: positions of two of them are given by the condition $e^{2}=e_{\mathrm{ex}(\mathrm{h})}^{2}(r ; a)$, where the function $e_{\mathrm{ex}(\mathrm{h})}^{2}(r ; a)$ is defined by the relation (9), location of the third extreme is given by condition

$e^{2}=e_{\mathrm{ex}(\mathrm{ph})}^{2}(r) \equiv \frac{3}{4} r$.

If the extreme determined by the condition (43) corresponds to a local minimum of the function $y_{\mathrm{ph}}(r ; a, e)$, this minimum lies between two local maxima given by the function 
$e_{\mathrm{ex}(\mathrm{h})}^{2}(r ; a)$. Since this function determines also extrema of the function of horizons $y_{\mathrm{h}}(r ; a, e)$, it follows that in a stationary region out of the black hole there are always two photon orbits playing a role of inner limit on the existence of circular orbits. The outer limit is then given by the outer static radius $r_{\mathrm{s} 2}$ (if $r_{\mathrm{s} 2}<r_{\mathrm{c}}$ ) or by the cosmological horizon $r_{\mathrm{c}}$. If the extreme given by the condition (43) corresponds to a local maximum, we denote it as $y_{\mathrm{ph}, \max }$, it is located under the inner black-hole horizon $r_{-}$. Then for the cosmological parameter $y_{\mathrm{h}, \text { min }}<y<y_{\mathrm{ph}, \text { max }}$ (if $y_{\mathrm{ph}, \max }<y_{\mathrm{h}, \max }$ ) or $y_{\mathrm{h}, \text { min }}<y<y_{\mathrm{h}, \text { max }}$ (if $y_{\mathrm{ph}, \text { max }}>y_{\mathrm{h}, \text { max }}$ ), there are other two photon orbits in the region between the inner static radius and the inner black-hole horizon, $r_{\mathrm{s} 1}<r<r_{-}$. Moreover, one of these photon orbits is stable against radial perturbations, see [42].

In naked-singularity spacetimes, in dependence of spacetime parameters, there can be up to four photon circular orbits. Such a situation takes place for those spacetime parameters $a$ and $e$, for which both the function $y_{\mathrm{h}}(r ; a, e)$ has local extremes with a minimum $y_{\mathrm{h}, \min }>0$, and the local extreme of the function $y_{\mathrm{ph}}(r ; a, e)$, given by the condition (43), corresponds to a local minimum - we denote it as $y_{\mathrm{ph}, \min }$. Then for $0<y<y_{\mathrm{h}, \text { min }}$ (if $y_{\mathrm{ph}, \min }<0$ ), or for $y_{\mathrm{ph}, \min }<y<y_{\mathrm{h}, \text { min }}$ (if $y_{\mathrm{ph}, \min }>0$ ) we get $\mathrm{KNdS}$ naked singularities with four circular photon orbits in the region between the static radii, $r_{\mathrm{s} 1}<r<r_{\mathrm{s} 2}$. Remaining naked-singularity spacetimes contain maximally two circular photon orbits located above the inner static radius. The upper limit for their existence is again given either by the outer static radius or by the cosmological horizon. If the local extreme given by the condition (43) corresponds to the local maximum $y_{\mathrm{ph} \text {, max }}$, then for $y>y_{\mathrm{ph}, \max }$ the KNdS spacetime contains no circular photon orbit in the equatorial plane. Behavior of the function $y_{\mathrm{ph}}(r ; a, e)$ for various values of spacetime parameters is presented in the Fig. 4.

For further discussion it is useful to distinguish between particular classes of equatorial circular photon orbits. The plus-family circular photon orbits are given by the condition

$$
\begin{aligned}
a & =a_{\mathrm{ph}(1,2)}^{(+)}(r ; y, e) \\
& \equiv \frac{\sqrt{r\left(1-y r^{3}\right)-e^{2}} \pm \sqrt{r\left(1-3 y r^{2}\right)-e^{2}\left(1-2 y r^{2}\right)}}{y r^{2}},
\end{aligned}
$$

while the minus-family circular photon orbits are given by the condition (for $a>0$ )

$$
\begin{aligned}
a & =a_{\mathrm{ph}}^{(-)}(r ; y, e) \\
& \equiv \frac{-\sqrt{r\left(1-y r^{3}\right)-e^{2}}+\sqrt{r\left(1-3 y r^{2}\right)-e^{2}\left(1-2 y r^{2}\right)}}{y r^{2}} .
\end{aligned}
$$

Circular photon orbits often form lower but in many cases also upper boundary of a region where circular test-particle orbits can exist. How such regions of existence for both plus and minus family orbits look like shows Fig. 5, in which solid curves correspond to the boundary given by photon orbits, dashed lines determine static radii and dotted curves determine horizons of corresponding KNdS spacetimes. Notice some interesting cases: (i) in the Fig. $5 \mathrm{c}$ we can see that there are naked-singularity spacetimes, in which the region of existence of circular orbits is split by a region where motion along equatorial circular orbits is impossible, (ii) it is shown that circular test-particle orbits can exist also in the nakedsingularity spacetimes with no photon circular orbits, see Fig. 5d, (iii) there are KNdS spacetimes [of both kind-naked singularities (Fig. 5a-f) and black holes (Fig. 5e)], in which the existence of equatorial circular orbits of both test particles and photons is impossible at all.

From behavior of the functions (44) and Fig. 5 follow that for given values of cosmological and charge parameters $y$ and $e$ allowing existence of equatorial circular orbits (see subsequent discussion), there is always a maximal value of rotational parameter

$a=a_{\max }^{(+)}(y, e) \equiv \frac{1}{16 y e^{3}}\left(3 \sqrt{3-32 y e^{4}}+\sqrt{27-256 y e^{6}}\right)$,

for which equatorial circular orbits belonging to the plusfamily can exist. ${ }^{2}$ From detailed analysis of the function $a_{\max }^{(+)}(y, e)$ follows that equatorial circular orbits exist only in those $\mathrm{KNdS}$ spacetimes whose cosmological parameter meet the condition

$y \leq \frac{3}{32 e^{4}}, \quad$ if $e^{2} \leq 9 / 8$,

or

$y \leq y_{\mathrm{ex}(\mathrm{s})}(e) \equiv \frac{27}{256 e^{6}}, \quad$ if $e^{2} \geq 9 / 8 ;$

the last condition is equivalent to the upper limit for the cosmological parameter enabling existence of static radii in given $\mathrm{KNdS}$ spacetime.

Limiting values of cosmological and charge parameters, for which circular orbits of the minus-family just (i.e. for $a=0$ ) disappear, are given by relations

$y=\frac{u-1}{2 u^{3}}, \quad e^{2}=\frac{u}{2}(3-u), \quad 1<u \leq 3$,

where $u$ is auxiliary parameter. Maximal values of both cosmological and charge parameters we get for $u=3 / 2$, giving values $y=2 / 27$ and $e^{2}=9 / 8$, which are the same as the limits for the existence of KNdS black holes. Note that for

\footnotetext{
2 This value corresponds to the maximum of the function $a_{\mathrm{ph}(1)}^{(+)}(r ; y, e)$, whose position is given by the relation (43).
} 
Fig. 4 Various profiles and positions of functions $y_{\mathrm{h}}(r ; a, e), y_{\mathrm{s}}(r ; e)$ and $y_{\mathrm{ph}}(r ; a, e)$ determining for given values of spacetime parameters $y, a, e$ locations of horizons (solid curve), static radii (dashed curve) and photon orbits (dotted curve)

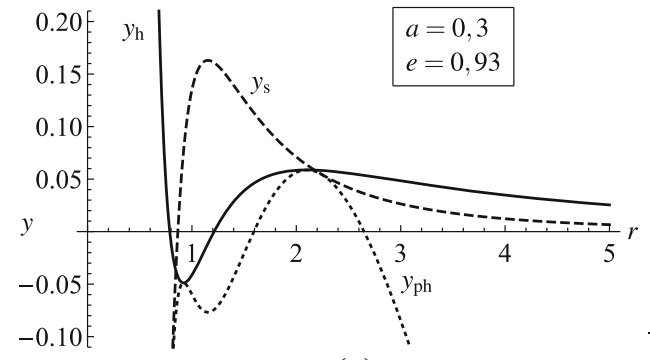

(a)

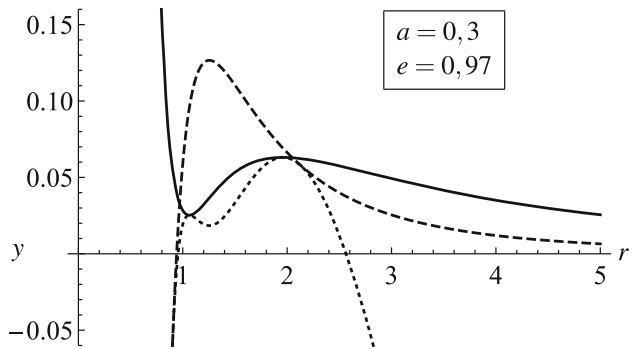

(b)
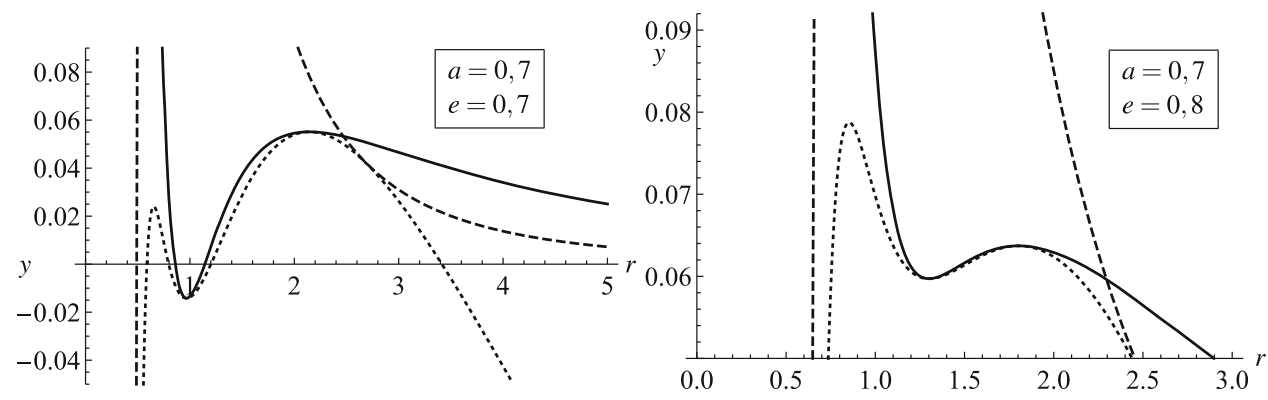

(c)

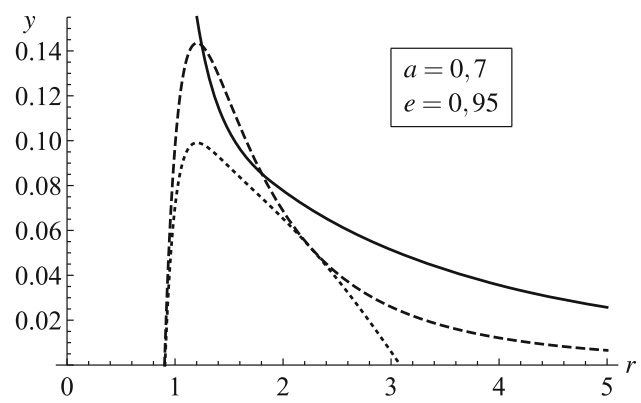

(e)

(d)

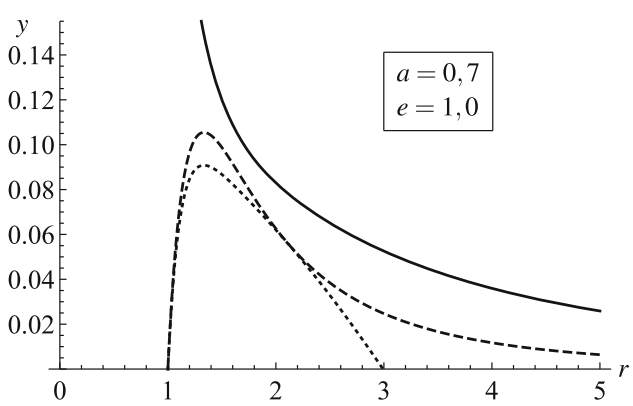

(f)

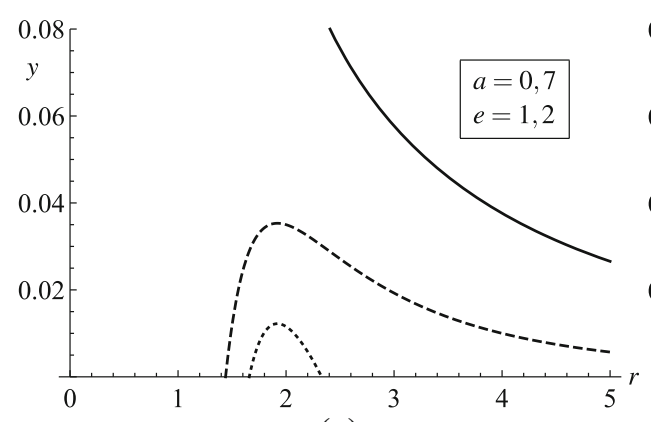

(g)

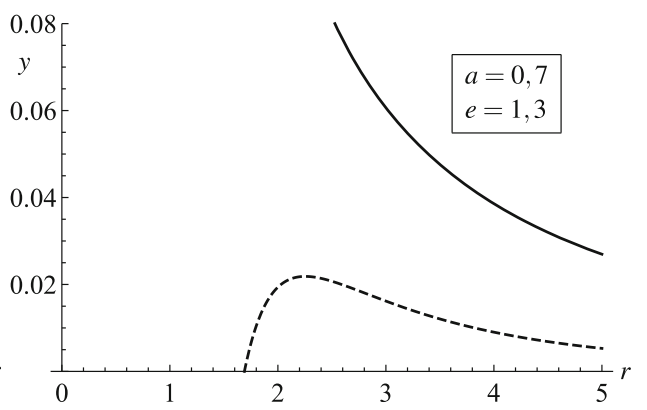

(h) $u=3$ we get $e=0$ and $y=1 / 27$, which is the limiting value of the cosmological parameter for the existence of minus-family equatorial circular orbits in the Kerr-de Sitter spacetime [30] and also for the existence of Schwarzschildde Sitter black holes [29].

\subsection{Orientation of circular orbits}

Like in the Kerr and Kerr-de Sitter spacetimes, also in the Kerr-Newman-de Sitter spacetime we distinguish prograde and retrograde circular motion of a particle with respect to the rotation of a central black hole/naked singularity. Particles rotating in the same direction as the center are called corotating, particles rotating in the opposite direction are called counterrotating.

In order to determine the direction of orbital motion, it is necessary to choose suitable reference frame in which azimuthal component of particle's 4-momentum is measured. Due to dragging of inertial frames, which takes place in all rotating spacetimes, the most suitable reference frame seems 


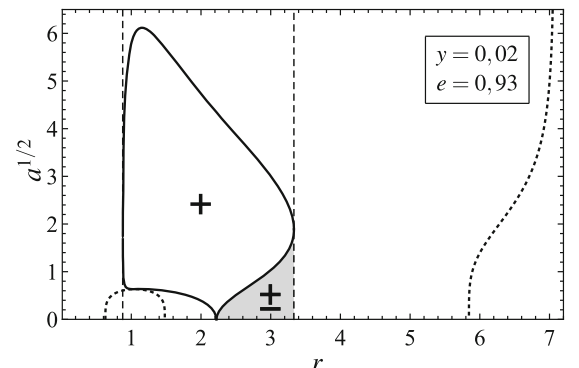

(a)

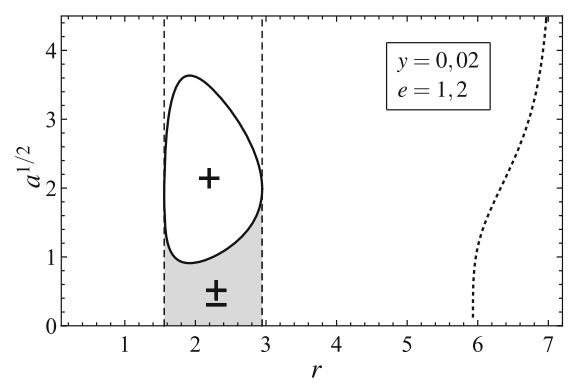

(d)

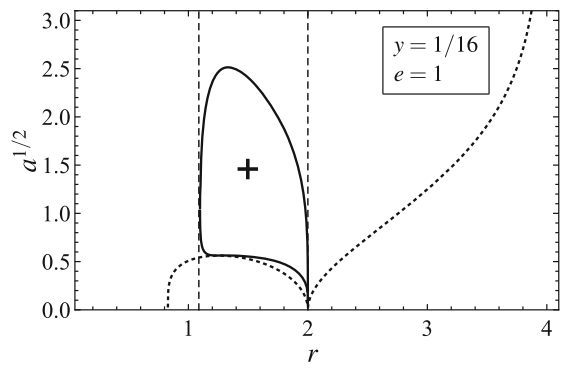

(b)

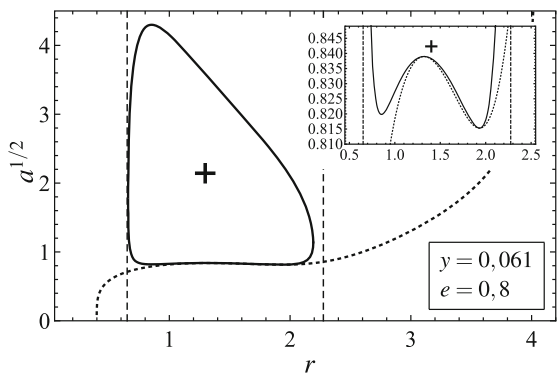

(e)

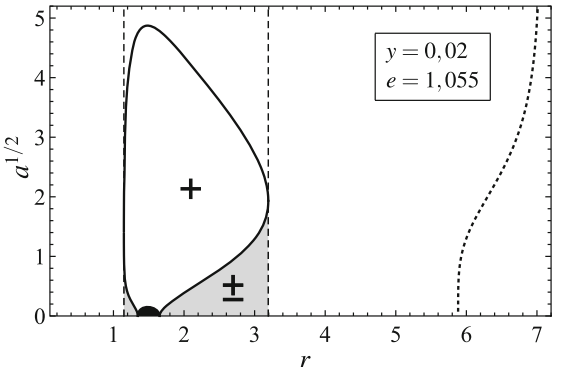

(c)

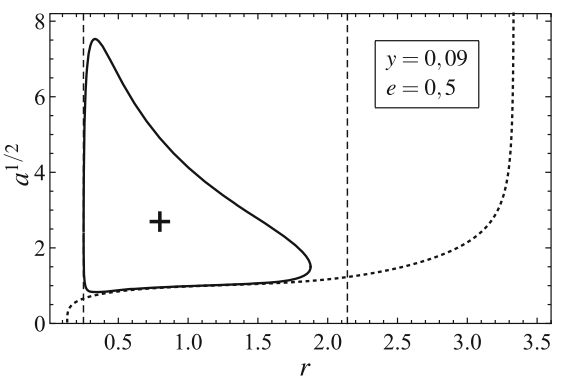

(f)
Fig. 5 Characteristic regions of existence of the equatorial circular orbits of the plus (+) and minus (-) families in the KNdS spacetimes. Solid curves correspond to circular photon orbits of a given family. Dashed lines and dotted curves determine static radii and horizons of

to be the locally non-rotating frame (LNRF) [46]. In the KNdS spacetime, the LNRF is determined by the tetrad of basis 1 -forms

$$
\begin{aligned}
& \boldsymbol{\omega}^{(t)}=\left(\frac{\Delta_{r} \Delta_{\theta} \varrho^{2}}{I^{2} \Sigma^{2}}\right)^{1 / 2} \mathbf{d} t, \\
& \omega^{(\varphi)}=\left(\frac{\Sigma^{2} \sin ^{2} \theta}{I^{2} \varrho^{2}}\right)^{1 / 2}(\mathbf{d} \varphi-\Omega \mathbf{d} t), \\
& \omega^{(r)}=\left(\frac{\varrho^{2}}{\Delta_{r}}\right)^{1 / 2} \mathbf{d} r, \\
& \omega^{(\theta)}=\left(\frac{\varrho^{2}}{\Delta_{\theta}}\right)^{1 / 2} \mathbf{d} \theta,
\end{aligned}
$$

where

$\Omega=\frac{a}{\Sigma^{2}}\left[\left(r^{2}+a^{2}\right) \Delta_{\theta}-\Delta_{r}\right]$

is the angular velocity $\mathrm{d} \varphi / \mathrm{d} t$ of the LNRF and

$\Sigma^{2}=\left(r^{2}+a^{2}\right)^{2} \Delta_{\theta}-a^{2} \Delta_{r} \sin ^{2} \theta$.

Locally measured components of particle's 4-momentum in the LNRF are given by its projection onto the LNRF-tetrad, $P^{(\alpha)}=\omega^{(\alpha)}(\mathbf{P})=\omega_{\mu}^{(\alpha)} P^{\mu}$, where $P^{\mu}=\mathrm{d} x^{\mu} / \mathrm{d} \lambda$ are coordinate components of particle's 4-momentum. For a test particle with rest mass $m, P^{\mu}=m \mathrm{~d} x^{\mu} / \mathrm{d} \tau \equiv m \dot{x}^{\mu}$, where $\tau$ is its proper time. The locally measured azimuthal component given KNdS spacetimes, respectively. Minus-family orbits exist only in the shaded regions $(\mathbf{a}, \mathbf{c}, \mathbf{d})$. In the black region $(\mathbf{c})$, there are no circular orbits

of particle's 4-momentum is, then, given by relation

$P^{(\varphi)}=\frac{m \Sigma}{I \rho}(\dot{\varphi}-\Omega \dot{t})$.

Using geodesic Eqs. (26)-(27) and restricting into the equatorial plane $\theta=\pi / 2$, we obtain for $\dot{t}$ and $\dot{\varphi}$ the expressions

$\dot{t}=\frac{I}{r^{2} \Delta_{r}}\left\{E\left[\left(r^{2}+a^{2}\right)^{2}-a^{2} \Delta_{r}\right]-a L\left[\left(r^{2}+a^{2}\right)-\Delta_{r}\right]\right\}$,

$\dot{\varphi}=\frac{I}{r^{2} \Delta_{r}}\left\{a E\left[\left(r^{2}+a^{2}\right)-\Delta_{r}\right]+L\left(\Delta_{r}-a^{2}\right)\right\}$,

and after adding them into (56) we finally get

$P^{(\varphi)}=\frac{m r}{\Sigma_{\pi / 2}} L$,

where $\Sigma_{\pi / 2} \equiv \Sigma(\theta=\pi / 2)$. We can see that the sign of locally measured azimuthal componet of 4-momentum of a particle on equatorial circular orbit is given by the sign of its specific angular momentum on such orbit. Orbits with $P^{(\varphi)}>0$, i.e. with $L>0$, are corotating, while the orbits with $P^{(\varphi)}<0$, i.e. with $L<0$, are counterrotating.

Equivalently, the orientation of equatorial circular orbit can be determined by comparison of angular velocity of a particle (so-called Keplerian angular velocity) $\Omega_{\mathrm{K}}=\mathrm{d} \varphi / \mathrm{d} t=$ $\dot{\varphi} / \dot{t}$, where $\dot{t}$ a $\dot{\varphi}$ are given by relations (57) and (58), with angular velocity of the LNRF on the same orbit. Substituting $E$ and $L$ in relations (57) and (58) by their profiles (37) and 
(38) for circular orbits, we get for each family of particles the radial profile of Keplerian angular velocity:

$\Omega_{\mathrm{K}} \pm= \pm \frac{1}{r^{2} / \sqrt{r\left(1-y r^{3}\right)-e^{2}} \pm a}$.

Radial profile of the LNRF angular velocity in the equatorial plane is given by relation (54) for $\theta=\pi / 2$ :

$\Omega_{\mathrm{LNRF}}=\frac{a\left[2 r+y r^{2}\left(r^{2}+a^{2}\right)-e^{2}\right]}{r^{2}\left(r^{2}+a^{2}\right)+a^{2}\left[2 r+y r^{2}\left(r^{2}+a^{2}\right)-e^{2}\right]}$

Then, a given orbit is corotating or conterrotating, if $\Omega_{\mathrm{K}}>$ $\Omega_{\mathrm{LNRF}}$ or $\Omega_{\mathrm{K}}<\Omega_{\mathrm{LNRF}}$ on given $r$, respectively.

Detailed analysis of the functions (38), describing behavior of specific angular momentum of particles on equatorial circular orbits, reveals that, like in the Kerr-de Sitter spacetime, also in the KNdS spacetime the minus-family orbits are always counterrotating. Plus-family orbits are mostly corotating but, as in the Kerr-de Sitter spacetime, there are counterrotaing plus-family orbits too. Similar behavior of plusfamily orbits have been found also in the Kerr $[47,48]$ and the Kerr-Newman $[49,50]$ spacetimes, but only in nakedsingularity spacetimes with sufficiently low value of their rotational parameter; in Kerr-de Sitter and KNdS spacetimes, counterrotating plus-family orbits can be found also around black holes.

Counterrotating plus-family orbits are separated from the corotating ones by orbits with zero angular momentum, $L=$ 0 , which are given by the condition

$$
\begin{aligned}
e^{2}= & e_{(L=0)}^{2}(r ; y, a) \\
\equiv & \frac{1}{2 a^{2}}\left\{-\left(r^{2}+a^{2}\right)^{2}+4 a^{2} r\left[1+\frac{1}{2} y r\left(r^{2}+a^{2}\right)\right]\right. \\
& \left.+\left(r^{2}+a^{2}\right) \sqrt{\left(r^{2}+a^{2}\right)^{2}-4 a^{2} r\left[1+y r\left(2 r^{2}+a^{2}\right)\right]}\right\} .
\end{aligned}
$$

Zero-points of the function (62) determine locations of the $L=0$ orbits in an uncharged Kerr-de Sitter spacetime. For $e^{2}>0$, the particular $\mathrm{KNdS}$ spacetime can contain up to four equatorial circular orbits with zero angular momentum. Note that in the Kerr-de Sitter spacetime there are maximally three such orbits [30].

In black-hole $\mathrm{KNdS}$ spacetimes, the plus-family orbits change their orientation from the corotating ones to the counterrotating ones always near the outer static radius. In some black-hole spacetimes, the plus-family orbits change their orientation also in the region under the inner horizon where the orbits nearby the singularity are counterrotating, above them are corotating orbits followed by the counterrotating ones. For higher values of charge parameter $e$ even all plusfamily orbits under the inner horizon of the KNdS black hole can be counterrotating.
In naked-singularity spacetimes, the plus-family orbits can change their orientation up to 4-times. This is the case of those naked singularities, which spacetime parameters are close to the values for the extreme KNdS black hole. Going from singularity to static radius, there are at first counterrotating plus-family orbits, followed by narrow region of corotating ones, which are replaced by also not very broad region of counterrotating orbits, after which there is a main region of corotating orbits which is replaced by relatively narrow region of counterrotating orbits near the static radius. For higher values of rotational and/or charge parameters, the inner region of spacetime near the singularity can contain only counterrotating plus-family orbits, thus, the plusfamily orbits change their orientation only twice (see Fig. 3b). Finally, there are also naked-singularity spacetimes allowing only counterrotating plus-family orbits.

\subsection{Stability of equatorial circular orbits}

Circular motion of a test particle is stable against radial perturbations if given orbit corresponds to a local minimum of the effective potential $V_{+}$. Equivalently, for stable circular orbits $\mathrm{d}^{2} \widetilde{R} / \mathrm{d} r^{2}<0 .{ }^{3}$ Transition between the stable and unstable orbits is formed by so-called marginally stable orbits. These are given by the condition $\mathrm{d}^{2} \widetilde{R} / \mathrm{d} r^{2}=0$, which must be satisfied together with conditions (36) for circular orbits, leading to the quadratic equation for rotational parameter $a$ :

$$
\begin{aligned}
& a^{2}\left[3 r-4 e^{2}+y r^{3}\left(1-4 y r^{3}\right)\right] \mp 8 a\left[r\left(1-y r^{3}\right)-e^{2}\right]^{3 / 2} \\
& -r^{2}\left[r-6-y r^{3}(4 r-15)\right]+4 e^{4}-3 e^{2} r\left(3-4 y r^{3}\right)=0,
\end{aligned}
$$

Its roots define functions

$$
\begin{aligned}
& a_{\mathrm{ms}(1,2)}^{(+)}(r ; y, e) \equiv \frac{4\left[r\left(1-y r^{3}\right)-e^{2}\right]^{3 / 2} \pm r \sqrt{\mathscr{D}}}{3 r-4 e^{2}+y r^{3}\left(1-4 y r^{3}\right)} \\
& a_{\mathrm{ms}(1,2)}^{(-)}(r ; y, e) \equiv \frac{-4\left[r\left(1-y r^{3}\right)-e^{2}\right]^{3 / 2} \pm r \sqrt{\mathscr{D}}}{3 r-4 e^{2}+y r^{3}\left(1-4 y r^{3}\right)},
\end{aligned}
$$

where

$$
\begin{aligned}
\mathscr{D}= & \left(1-4 y r^{3}\right)\left\{3 y^{2} r^{5}\left(5 r-4 e^{2}\right)\right. \\
& -y r\left[4 e^{4}-3 e^{2} r(4 r+3)+2 r^{2}(5 r+3)\right] \\
& \left.+3 r^{2}-2 r-e^{2}(4 r-3)\right\} .
\end{aligned}
$$

\footnotetext{
${ }^{3}$ For the stability of circular geodesics against latitudinal (vertical) perturbations see [16,51]. It follows that all circular geodesics, being stable against radial perturbations, are also stable against the latitudinal ones.
} 


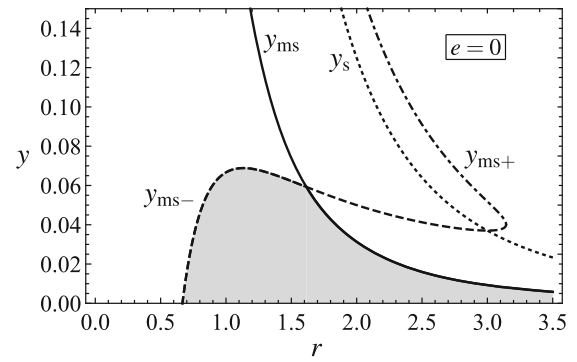

(a)

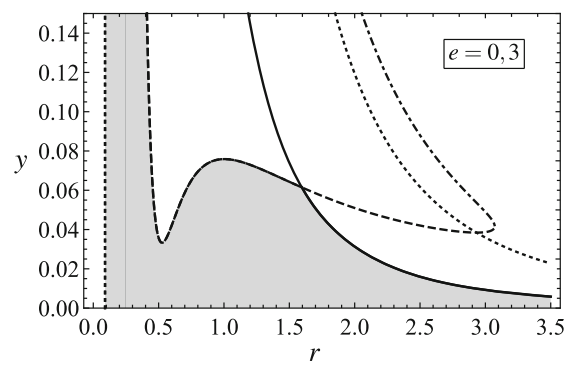

(d)

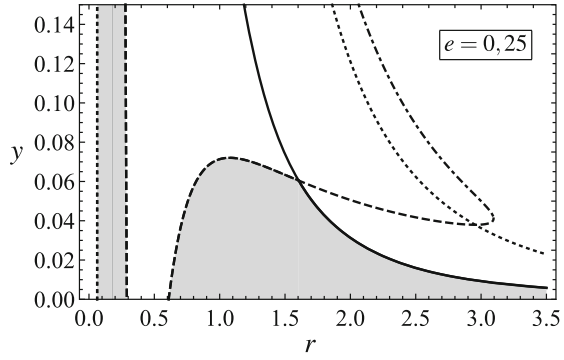

(b)

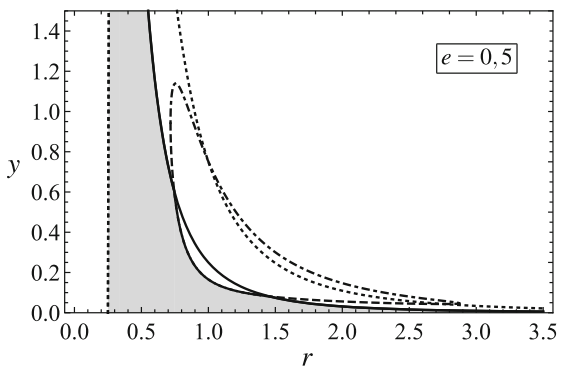

(e)

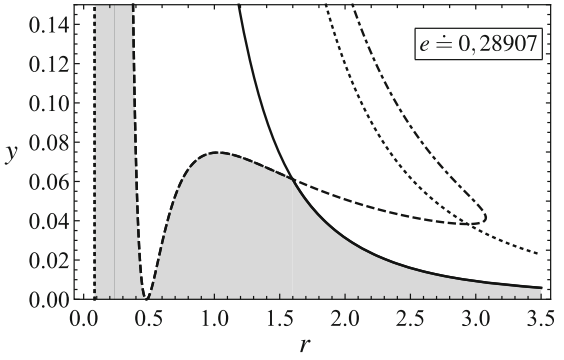

(c)

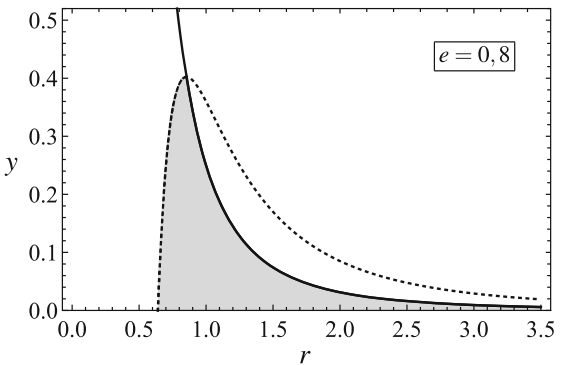

(f)
Fig. 6 Various forms of stability regions (shaded), enabling the existence of stable equatorial circular orbits in the KNdS spacetimes, in dependence of a charge parameter $e$. The regions are given by common intersection of conditions (67)-(69) for the cosmological parameter $y$. For $e \gtrsim 0,55548$, the stability region is limited only by the functions $y_{\mathrm{s}}(r ; e)$ and $y_{\mathrm{ms}}(r)$
Regions, where functions $a_{\mathrm{ms}(1,2)}^{( \pm)}(r ; y, e)$ are real, determine regions, in which motion along stable equatorial circular orbits is possible. More precisely, stable circular orbits exist in those KNdS spacetimes which cosmological parameter $y$ satisfies simultaneously the conditions

$y \leq y_{\mathrm{s}}(r ; e) \equiv \frac{r-e^{2}}{r^{4}}$,

$y \leq y_{\mathrm{ms}}(r) \equiv \frac{1}{4 r^{3}}$,

$y \leq y_{\mathrm{ms}-}(r ; e), \quad$ or $\quad y \geq y_{\mathrm{ms}+}(r ; e)$,

where

$$
\begin{aligned}
& y_{\mathrm{ms} \pm}(r ; e) \equiv \frac{1}{6 r^{4}\left(5 r-4 e^{2}\right)}\left\{4 e^{4}-3 e^{2} r(4 r+3)\right. \\
& +2 r^{2}(5 r+3) \pm 2\left[4 e^{8}-6 e^{6} r(4 r+3)-12 e^{4} r^{3}(4 r-3)\right. \\
& \left.\left.\quad+3 e^{2} r^{3}\left(12 r^{2}-50 r-9\right)-r^{4}\left(20 r^{2}-60 r-9\right)\right]^{1 / 2}\right\} .
\end{aligned}
$$

Detailed analysis of conditions (67)-(69) reveals that maximal value of the cosmmological parameter allowing motion along stable circular geodesics of the KNdS spacetime with a charge parameter $e>0$ corresponds, independetly of the rotational parameter $a$, to extremal value of the function $y_{\mathrm{s}}(r ; e)$, i.e. to

$y_{\text {stab, } \max }=y_{\mathrm{ex}(\mathrm{s})} \equiv \frac{27}{256 e^{6}}$.
For further discussion, the existence of local extrema of the function $y_{\mathrm{ms}-}(r ; e)$ is important. If $e=0$, the function $y_{\mathrm{ms}-}(r ; e)$ has one local extreme - maximum in the region satisfying condition (68), which value $y_{\text {stab, max }}^{(\mathrm{KdS})} \doteq 0,06886$ corresponds to maximal value of the cosmological parameter $y$ allowing existence of stable equatorial circular orbits in the Kerr-de Sitter spacetime [30]. If $0<e \lesssim 0,33202$, the function $y_{\mathrm{ms}-}(r ; e)$ has two local extrema, minimum $y_{\mathrm{ms}, \min }$ and maximum $y_{\mathrm{ms} \text {, max }}$, which values depend on the charge parameter $e$. For $e \lesssim 0,28907$, the $y_{\mathrm{ms} \text {, min }}$ is negative, while for higher values of $e$ it is positive. The other extreme $y_{\mathrm{ms}, \max }$ is always positive. For $y \in\left(y_{\mathrm{ms}, \min }, y_{\mathrm{ms}, \max }\right)$, the corresponding KNdS spacetime contains two separate regions of stable circular orbits. Influence of the charge parameter $e$ on character of regions with stable equatorial circular orbits in the KNdS spacetime is shown in the Fig. 6.

For given values of spacetime parameters $(y, a, e)$, the locations of marginally stable circular orbits are implicitly determined even by relations $a=a_{\mathrm{ms}(1,2)}^{(+)}(r ; y, e)$ valid for the plus-family orbits, or $a=a_{\mathrm{ms}(1,2)}^{(-)}(r ; y, e)$ valid for the minus-family ones. In subsequent discussion we restrict on the $a>0$ cases only. Detailed analysis of behavior of the functions (64) and (65) shows that in black-hole spacetimes allowing motion along stable equatorial circular orbits of a given family, there are for each family, in general, two marginally stable orbits, $r_{\mathrm{ms}(\mathrm{i})}$ and $r_{\mathrm{ms}(\mathrm{o})}$, located above the outer black-hole horizon. Stable circular orbits lie in the 


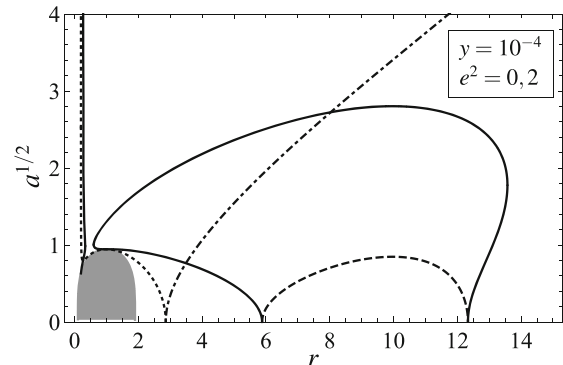

(a)

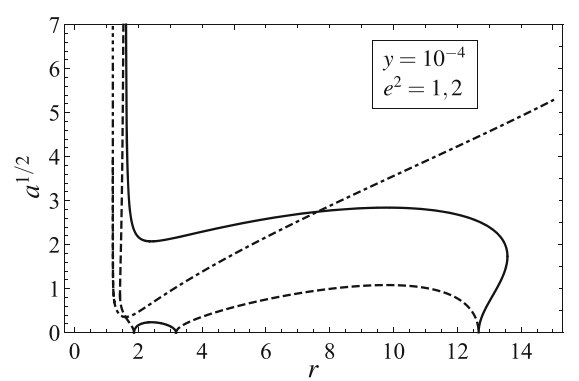

(d)

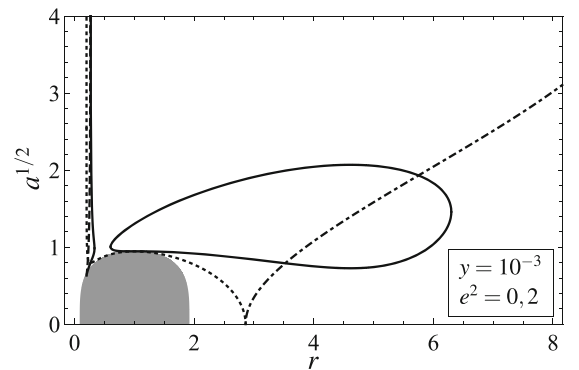

(g)

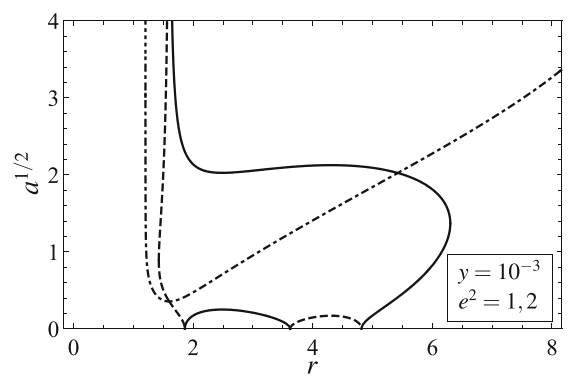

(j)

Fig. 7 Characteristic mutual positions of marginally stable and photon orbits in dependence of the rotational parameter $a$ for appropriately chosen KNdS spacetimes with cosmological parameter $y$ and charge parameter $e$. Plus/minus-family marginally stable orbits are given by

region between the inner and outer marginally stable orbits, $r_{\mathrm{ms}(\mathrm{i})}<r<r_{\mathrm{ms}(\mathrm{o})}$, while the unstable orbits are located in regions $r<r_{\mathrm{ms}(\mathrm{i})}$ and $r>r_{\mathrm{ms}(\mathrm{o})}$. The inner region of unstable orbits is extended down to the (inner) photon orbit of given family, while a boundary of the outer region of unstable orbits is formed either by the outer static radius or by

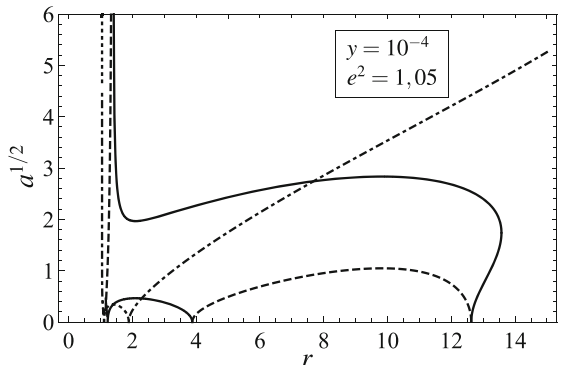

(c)

(b)

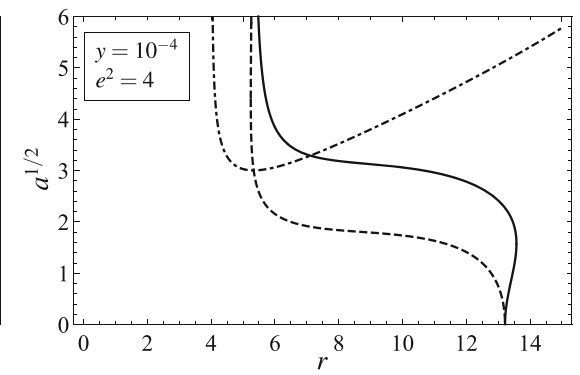

(f)

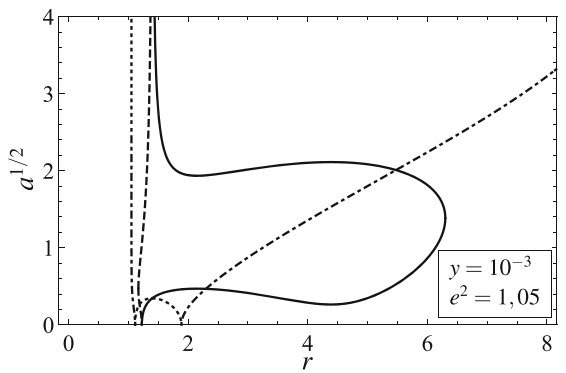

(i) solid/dashed curves, while the plus/minus-family photon orbits are determined by dotted/dashed-dotted curves. Shaded are the regions in between the inner and outer black-hole horizons, if they exist

the (outer) photon orbit (the latter is valid only for the plusfamily orbits in the KNdS spacetimes with no minus-family orbits.

In naked-singularity spacetimes allowing motion along stable equatorial circular orbits of a given family, there are for each family, in dependence of spacetime parameters, up 


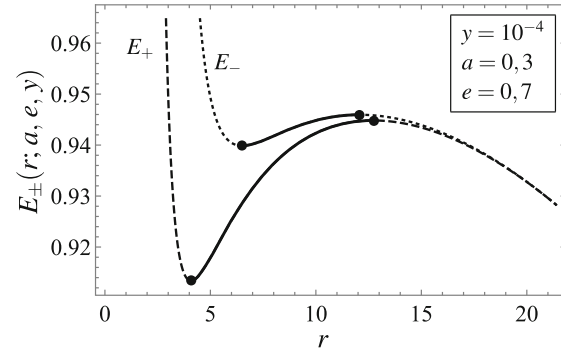

(a)

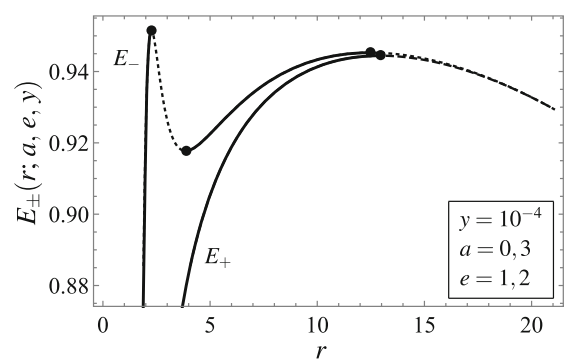

(d)

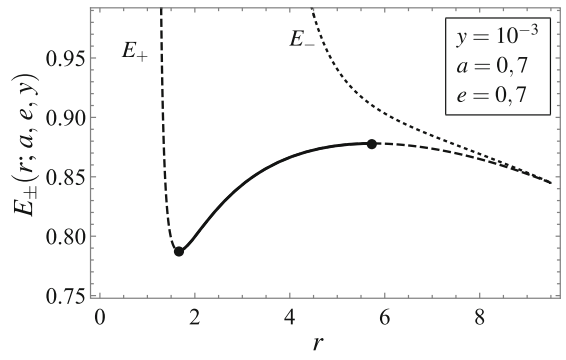

(b)

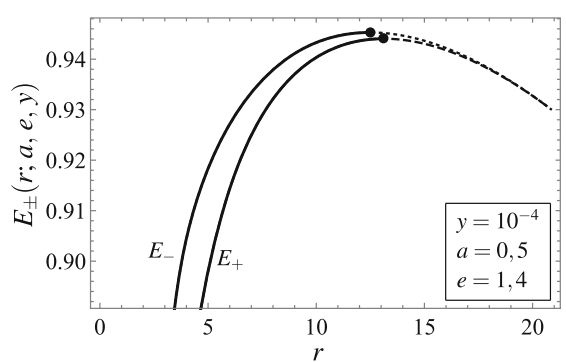

(e)

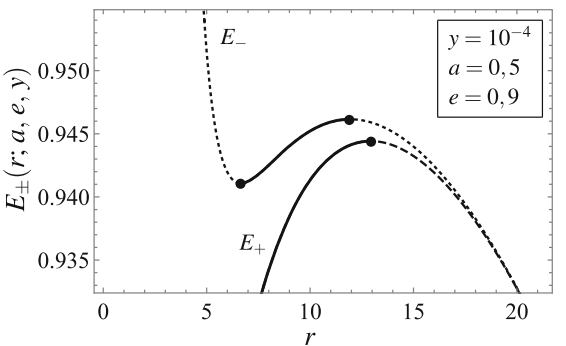

(c)

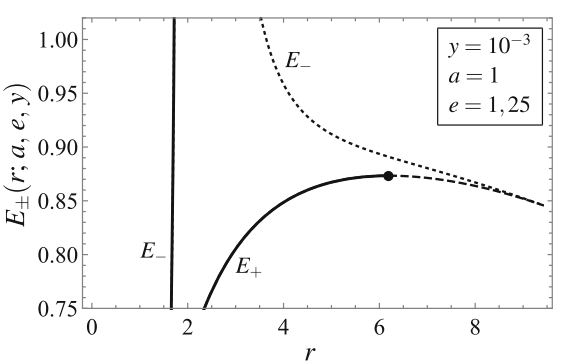

(f)
Fig. 8 Characteristic specific energy profiles of test particles on equatorial circular orbits of the plus/minus-family in the field of KNdS black holes $(\mathbf{a}-\mathbf{b})$ and naked singularities $(\mathbf{c}-\mathbf{f})$. Solid parts of the curves cor- respond to the motion along stable circular orbits of a given family, dashed and dotted parts represent unstable circular orbits. Solid circles denote marginally stable orbits of a given family to three marginally stable circular orbits. If there is only one marginally stable orbit (located at $r=r_{\mathrm{ms}}$ ), it separates the inner region of stable orbits $\left(r<r_{\mathrm{ms}}\right)$ from the outer region of unstable ones $\left(r>r_{\mathrm{ms}}\right)$. In the case of two marginally stable orbits, $r_{\mathrm{ms}(\mathrm{i})}$ and $r_{\mathrm{ms}(\mathrm{o})}$, the situation is similar to the one for black holes: the region of unstable orbits, $r<r_{\mathrm{ms}}(\mathrm{i})$, is followed by the region of stable orbits, $r_{\mathrm{ms}(\mathrm{i})}<r<r_{\mathrm{ms}(\mathrm{o})}$, above which there is again the region of unstable orbits, $r>r_{\mathrm{ms}(\mathrm{o})}$. If the spacetime possesses three marginally stable orbits of a given family, $r_{\mathrm{ms}(\mathrm{i})}, r_{\mathrm{ms}(\mathrm{c})}$ a $r_{\mathrm{ms}(\mathrm{o})}$, it contains two regions of stable orbits, $r<r_{\mathrm{ms}(\mathrm{i})}$ a $r_{\mathrm{ms}(\mathrm{c})}<r<r_{\mathrm{ms}(\mathrm{o})}$ and two regions of unstable ones, $r_{\mathrm{ms}(\mathrm{i})}<r<r_{\mathrm{ms}(\mathrm{c})}$ a $r>r_{\mathrm{ms}(\mathrm{o})}$. Outer boundary of unstable orbits is like in blackhole backgrounds formed either by the outer static radius or by the outer photon orbit. Analogically, the inner boundary of orbits (both unstable and stable) is formed either by the inner static radius or by the inner photon orbit. This is true also in the case when the innermost region of orbits is stable, but only for the plus-family orbits; stable minus-family orbits cannot end "at" the inner photon orbit. Nevertheless, there are naked-singularity spacetimes with the inner region of stable minus-family orbits bounded from the outer side by the inner photon orbit and from the inner side by the inner static radius, without crossing any marginally stable orbit.

Figure 7 shows mutual positions of marginally stable and photon orbits in dependence of the rotational parameter $a$ for selected KNdS spacetimes with prescribed cosmological and charge parameters $y$ and $e$. Note that the marginally stable minus-family orbit located between two minus-family photon orbits is unphysical because in this region the condition (40) is not valid and, thus, in this region no minus-family orbits exist.

Following the definition of marginally stable orbits, it is clear that these orbits must correspond to local extrema in radial profiles of the specific energy and specific angular momentum of particles on equatorial circular orbits given by the relations (37) and (38). Raising parts of the functions $E_{ \pm}(r ; a, e, y)$ correspond to stable circular orbits while descending parts correspond to unstable ones. Several interesting profiles, showing possible regions of stable circular orbits (which were discussed previously) and corresponding decrease of specific energy, are presented in the Fig. 8. If the inner boundary of stable plus-family orbits is given by the stable photon orbit, the specific energy of particles moving along orbits near the inner boundary decrease deeply to negative values and in the limit $r \rightarrow r_{\text {ph }}$ the specific energy $E_{+} \rightarrow-\infty$. Simultaneously, these orbits are, at least close to the inner boundary, counterrotating, which means that their specific angular momentum is also negative, and in the limit $r \rightarrow r_{\mathrm{ph}}, L_{+} \rightarrow-\infty$. Such a behavior of constants of motion $E_{+}$and $L_{+}$is typical for a wide range of nakedsingularity spacetimes of the Kerr-Newman type (with properly tuned parameters). For a more detailed discussion see [50], where the authors discussed also an efficiency of accretion in the Keplerian regime and anticipated an unstability of given naked singularity which behaves like apparently inex- 
haustible source of energy. Clearly, in order to obtain deeper insight it is necessary to study the evolution of naked singularity with respect to change of their parameters caused by the accretion of matter (see, e.g., [52]), which together with negative energy brings also negative angular momentum. For ultrahigh-energy particle collisions and optical effects in such a special Kerr-Newman naked-singularity background see also [53].

\section{Conclusions}

The paper brings the analysis of common influence of the electric charge, spacetime rotation and cosmic repulsion on properties of equatorial circular orbits of uncharged test particles, i.e. geodesics, in the frame of exact Kerr-Newmande Sitter solution of Einstein-Maxwell equations of General Relativity. The study was done for both the black-hole and naked-singularity backgrounds in a similar manner as for the Kerr-de Sitter spacetimes [30] and, in that sense, it extends our previous studies. Note that the separate analysis of common influence of the cosmic repulsion and electric charge on circular geodesics of the Reissner-Nordström-de Sitter spacetimes was done in [41]. In our final study, the crucial point is, therefore, the incorporation of all the three effects.

Influence of the cosmic repulsion, connected with a positive cosmological constant $\Lambda$, is reflected mainly in the existence of the outer boundary for general equatorial circular orbits (the outer static radius), and also for stable circular orbits (the outer marginally stable orbit), while the influence of spacetime rotation is connected with the existence of two families of orbits, and with various properties of each family.

Influence of the electric charge can be summarized in several points:

1. Together with the outer static radius, there is also the inner static radius connected with a charge repulsion, which limits the existence of equatorial circular orbits from the inner side.

2. Charge parameter of the KNdS spacetime determines maximal value of the cosmological parameter allowing existence of equatorial circular orbits.

3. Charge parameter of the KNdS spacetime determines maximal value of the cosmological parameter allowing existence of stable equatorial circular orbits.

4. Due to the existence of the stable photon circular orbit in the $\mathrm{KNdS}$ spacetime, there are naked-singularity spacetimes in which the innermost stable circular orbit of a test particle approaches this photon orbit, being characterized by the diverging specific energy and specific angular momentum, both going to $-\infty$.
The results presented in the paper are of general character, and in that sense they can be used in various subsequent analyses. Some sort of them can be found in recently published review paper [54]. Especially, discussion of naked singularities enables to show differences between black holes and even more compact objects like superspinars $[55,56]$, which existence follows from alternative theories of gravity. In near future, we plan to study properties of Keplerian discs orbiting KNdS black holes and naked singularities.

Acknowledgements The authors acknowledge support from the Research Centre of Theoretical Physics and Astrophysics, being a part of the Institute of Physics of the Silesian University in Opava, Czech Republic.

Data Availability Statement This manuscript has no associated data or the data will not be deposited. [Authors' comment: This is a theoretical work. There are no external and experimental data associated with the manuscript.]

Open Access This article is licensed under a Creative Commons Attribution 4.0 International License, which permits use, sharing, adaptation, distribution and reproduction in any medium or format, as long as you give appropriate credit to the original author(s) and the source, provide a link to the Creative Commons licence, and indicate if changes were made. The images or other third party material in this article are included in the article's Creative Commons licence, unless indicated otherwise in a credit line to the material. If material is not included in the article's Creative Commons licence and your intended use is not permitted by statutory regulation or exceeds the permitted use, you will need to obtain permission directly from the copyright holder. To view a copy of this licence, visit http://creativecomm ons.org/licenses/by/4.0/.

Funded by $\mathrm{SCOAP}^{3}$.

\section{References}

1. A.G. Riess et al., Astronom. J. 116, 1009 (1998)

2. S. Perlmutter et al., Astrophys. J. 517(2), 565 (1999)

3. D.O. Jones et al., Astrophys. J. 881(1), 19 (2019)

4. P.A.R. Ade, N. Aghanim, M. Arnaud et al., Planck Collaboration. Astronom. Astrophys. 594, 13 (2016)

5. E. Aubourg, S. Bailey, J.E. Bautista et al., BOSS Collaboration. Phys. Rev. D 92, 123516 (2015)

6. Y.B. Zel'dovich, I.D. Novikov, Relativistic Astrophysics 2: The Structure and Evolution of the Universe (University of Chicago Press, Chicago, 1983)

7. Ø. Grøn, S. Hervik, Einstein's General Theory of Relativity: With Modern Applications in Cosmology (Springer, New York, 2007)

8. B. Ratra, P.J.E. Peebles, Phys. Rev. D 37(12), 3406 (1998)

9. R.R. Caldwell, R. Dave, P.J. Steinhardt, Phys. Rev. Lett. 80(8), 1582 (1998)

10. E.J. Copeland, M. Sami, S. Tsujikawa, Int. J. Mod. Phys. D 15, $1753(2006)$

11. M.P. Dabrowski, M.A. Hendry, Astrophys. J. 498(1), 67 (1998)

12. Y.C. Ong, S.S. Hashemi, R. An, B. Wang, Eur. Phys. J. C 78(5), $405(2018)$

13. E. Kopteva, I. Bormotova, M. Churilova, Z. Stuchlík, Astrophys. J. 887(1), 98 (2019)

14. Carter, B.: In: Witt, C.D., Witt, B.S.D. (eds.) Black Holes, p. 57. Gordon and Breach, New York (1973) 
15. G.W. Gibbons, S.W. Hawking, Phys. Rev. D 15, 2738 (1977)

16. Z. Stuchlík, Bull. Astronom. Inst. Czechoslovakia 34(3), 129 (1983)

17. V. Faraoni, M. Lapierre-Léonard, A. Prain, J. Cosmol. Astropart. Phys. 10, 013 (2015)

18. V. Faraoni, Phys. Dark Universe 11, 11 (2016)

19. Z. Stuchlík, P. Slaný, S. Hledík, Astronom. Astrophys. 363(2), 425 (2000)

20. P. Slaný, Z. Stuchlík, Classical Quantum Gravity 22, 3623 (2005)

21. Z. Stuchlík, Mod. Phys. Lett. A 20(8), 561 (2005)

22. Z. Stuchlík, P. Slaný, J. Kovář, Class. Quantum Gravity 26(21), 215013 (2009). (34pp)

23. M.T. Busha, F.C. Adams, R.H. Wechsler, A.E. Evrard, Astrophys. J. 596, 713 (2003)

24. Z. Stuchlík, J. Schee, J. Cosmol. Astropart. Phys. 9, 018 (2011)

25. V. Pavlidou, N. Tetradis, T.N. Tomaras, J. Cosmol. Astropart. Phys. 05, 017 (2014)

26. Z. Stuchlík, S. Hledík, J. Novotný, Phys. Rev. D 94(10), 103513 (2016)

27. Z. Stuchlík, J. Schee, B. Toshmatov, J. Hladík, J. Novotný, J. Cosmol. Astropart. Phys. 06, 056 (2017)

28. A. Giusti, V. Faraoni, Phys. Dark Universe 26, 100353 (2019)

29. Z. Stuchlík, S. Hledík, Phys. Rev. D 60(4), 044006 (1999)

30. Z. Stuchlík, P. Slaný, Phys. Rev. D 69, 064001 (2004)

31. G.V. Kraniotis, Class. Quantum Gravity 21(19), 4743 (2004)

32. G.V. Kraniotis, Class. Quantum Gravity 22(21), 4391 (2005)

33. Z. Stuchlík, J. Kovář, Int. J. Mod. Phys. D 17, 2089 (2008)

34. M. Sereno, Phys. Rev. D 77(4), 043004 (2008)

35. E. Hackmann, C. Lämmerzahl, V. Kagramanova, J. Kunz, Phys. Rev. D 81(4), 044020 (2010)

36. D. Charbulák, Z. Stuchlík, Eur. Phys. J. C 77(12), 897 (2017)

37. Z. Stuchlík, D. Charbulák, J. Schee, Eur. Phys. J. C 78(3), 180 (2018)
38. R. Abuter, A. Amorim, M. Bauböck et al., GRAVITY Collaboration. Astronom. Astrophys. 618, L10 (2018)

39. M. Zajaček, A. Tursunov, A. Eckart, S. Britzen, Monthly Not. R. Astronom. Soc. 480(4), 4408 (2018)

40. M. Zajaček, A. Tursunov, A. Eckart, S. Britzen, E. Hackmann, V. Karas, Z. Stuchlík, B. Czerny, J.A. Zensus, J. Phys. Conf. Ser. 1258, 012031 (2019)

41. Z. Stuchlík, S. Hledík, Acta Phys. Slovaca 52(5), 363 (2002)

42. Z. Stuchlík, S. Hledík, Class. Quantum Gravity 17(21), 4541 (2000)

43. Z. Stuchlík, M. Calvani, Gen. Relat. Gravit. 23, 507 (1991)

44. C.W. Misner, K.S. Thorne, J.A. Wheeler, Gravitation (Freeman, San Francisco, 1973)

45. B. Carter, Phys. Rev. 174, 1559 (1968)

46. J.M. Bardeen, W.H. Press, S.A. Teukolsky, Astrophys. J. 178, 347 (1972)

47. Z. Stuchlík, Bull. Astronom. Inst. Czechoslovakia 31(3), 129 (1980)

48. D. Pugliese, H. Quevedo, R. Ruffini, Phys. Rev. D 84(4), 044030 (2011)

49. D. Pugliese, H. Quevedo, R. Ruffini, Phys. Rev. D 88(2), 024042 (2013)

50. M. Blaschke, Z. Stuchlík, Phys. Rev. D 94(8), 086006 (2016)

51. J. Bičák, Z. Stuchlík, Bull. Astronom. Inst. Czechoslovakia 27(3), 129 (1976)

52. Z. Stuchlík, Bull. Astronom. Inst. Czechoslovakia 32(2), 68 (1981)

53. Z. Stuchlík, M. Blaschke, J. Schee, Phys. Rev. D 96(10), 104050 (2017)

54. Z. Stuchlík, M. Kološ, J. Kovář, P. Slaný, A. Tursunov, Universe 6(2), $26(2020)$

55. E.G. Gimon, P. Hořava, Phys. Lett. B 672, 299 (2009)

56. Z. Stuchlík, J. Schee, Class. Quantum Gravity 27(21), 215017 (2010) 